\title{
Avaliação clínica e da espessura cutânea um ano após preenchimento de ácido hialurônico
}

\author{
Clinical and dermal thickness assessment one year after hyaluronic acid filler
}

\section{treatment}

\author{
Alessandra Grassi Salles ${ }^{1}$ \\ Adelina Fátima do \\ Nascimento Remigio ${ }^{2}$ \\ VAleria Berton Liguori \\ $\mathrm{ZACCHI}^{3}$ \\ Osmar Cássio Saito ${ }^{4}$ \\ Marcus Castro Ferreira 5
}

Trabalho realizado no Hospital das Clínicas da Faculdade de Medicina da Universidade de São Paulo, São Paulo, SP, Brasil.

Artigo submetido pelo SGP (Sistema de Gestão de Publicações) da RBCP.

Artigo recebido: $26 / 11 / 2010$ Artigo aceito: 5/2/2011

\begin{abstract}
RESUMO
Introdução: Poucos estudos avaliam a durabilidade dos produtos de preenchimento facial de forma objetiva e não-invasiva. O objetivo deste estudo foi avaliar a durabilidade de formulação de ácido hialurônico (Derma Hyal®) no preenchimento de sulco nasogeniano, comparando percepção do efeito clínico e espessura de partes moles medida por ultrassonografia. Método: Dez mulheres foram tratadas. Avaliação foi realizada após um, três, seis, nove e doze meses, clinicamente e por ultrassonografia. Resultados: A média da espessura à ultrassonografia foi $0,38 \pm 0,14$ no pré, $0,69 \pm 0,19$ após 1 mês, $0,65 \pm 0,17$ após 3 meses, $0,61 \pm 0,22$ após 6 meses, $0,57 \pm 0,23$ após 9 meses e $0,55 \pm 0,14$ após 12 meses. Os dados analisados pelo teste Friedman não se mostraram estatisticamente significativos. Em relação à satisfação, aos 6 meses, 3 pacientes consideraram o resultado insatisfatório, 5 , pouco satisfatório e 2, satisfatório. Apenas uma paciente considerou o resultado satisfatório aos 9 meses. Aos 12 meses, uma considerou pouco satisfatório e as demais, insatisfatório. Todas responderam que realizariam novamente o procedimento. Conclusões: A ultrassonografia demonstrou ser método objetivo e não-invasivo na avaliação da durabilidade de preenchimento, desde que avaliado com correlação clínica. Demonstrou-se aumento da espessura cutânea até 12 meses após injeção em sulco nasogeniano (SNG), com decréscimo progressivo. Embora exista diferença dos valores da ultrassonografia ao longo do tempo, essas não foram estatisticamente significantes. Clinicamente, o efeito percebido pelas pacientes se deu até o nono mês após aplicação do produto avaliado.
\end{abstract}

Descritores: Ácido hialurônico. Ultrassonografia. Face.

\begin{abstract}
Background: Few scientific studies evaluate the durability of existent fillers with objective parameters and non invasive methods. The objective of this study was to evaluate durability of a hyaluronic acid formula (Derma Hyal ${ }^{\circledR}$ ) in the nasolabial fold treatment, comparing the perception of clinical effect and soft tissue thickness measured by ultrasound. Methods: Ten women were treated. Evaluation was made before, after 1, 3, 6, 9 and 12 months, clinically and by ultrasound. Results: Median ultrasound thickness was $0.38 \pm 0.14$ pre, $0.69 \pm 0.19$ after 1 month, $0.65 \pm 0.17$ after 3 months, $0.61 \pm 0.22$ after 6 months, $0,57 \pm 0.23$ after 9 months and $0.55 \pm 0.14$ after 12 months. Data analyzed by Friedman's test were not statistically significant. Regarding patient satisfaction after 6 months, 3 considered the results unsatisfactory, 5 fairly satisfactory and 2 satisfactory. Only one patient considered the treatment satisfactory at 9 months. By 12 months one considered it fairly satisfactory and nine unsatisfactory. All patients said they would do treatment again in another opportunity. Conclusions: Ultrasound evaluation of dermal thickness after filler treatment proved to be an objective and non invasive method, since when associated with clinical examination. Increase in soft tissue thickness was demonstrated until 12 months after nasolabial fold injection, with progressive decrease after this period. Despite the increase in soft tissue thickness at ultrasound, it was not statistically significant. Clinical effect was noticed by patients until nine months after treatment with this product.
\end{abstract}

Keywords: Hyaluronic acid. Ultrasonography. Face.

1. Doutora em Cirurgia Plástica; Coordenadora do Grupo de Cosmiatria da Disciplina de Cirurgia Plástica da Faculdade de Medicina da Universidade de São Paulo (FMUSP), São Paulo, SP, Brasil.

2. Membro Titular da Sociedade Brasileira de Cirurgia Plástica (SBCP); Médica pesquisadora da Disciplina de Cirurgia Plástica da FMUSP, São Paulo, SP, Brasil.

3. Membro Especialista da SBCP, Médica Pesquisadora da Disciplina de Cirurgia Plástica da FMUSP, São Paulo, SP, Brasil.

4. Doutor em Radiologia; Assistente do Instituto de Radiologia do Hospital das Clínicas da FMUSP, São Paulo, SP, Brasil.

5. Professor Titular da Disciplina de Cirurgia Plástica da FMUSP; Chefe do Serviço de Cirurgia Plástica do Hospital das Clínicas da FMUSP, São Paulo, SP, Brasil. 


\section{INTRODUÇÃO}

O ácido hialurônico é um polissacarídeo glicosaminoglicano presente na matriz extracelular da pele, tecido conectivo e no humor vítreo. Tem como funções hidratação, lubrificação e estabilização desses meios ${ }^{1,2}$. Representa uma alternativa no tratamento do envelhecimento facial e tem sido utilizado há mais de uma década no preenchimento de partes moles para corrigir depressões, rugas e sulcos. O comportamento biológico é bem conhecido, com estudos histológicos, sendo absorvido gradativamente ao longo dos meses ${ }^{3-6}$. Suas potenciais complicações são infrequentes e autolimitadas e incluem reações inflamatórias, pequenos hematomas e/ou equimoses, abscessos nos sítios de aplicação, necrose tecidual (por injeção intravascular ou compressão da rede vascular adjacente), edema persistente e granulomas ${ }^{1,6}$. Edema persistente e granulomas podem ser causados por alergia ao material ou por resposta imunológica aos componentes protéicos presentes nas preparações de ácido hialurônico, e podem ser tratados com injeção local de hialuronidase $\mathrm{e}^{2,7}$.

Atualmente, há grande número de apresentações comerciais disponíveis; entretanto, há poucos estudos científicos de longo prazo que comprovem a real durabilidade de cada produto, ou que forneçam comparações entre os mesmos ${ }^{8-10}$.

A ultrassonografia, utilizada previamente em vários estudos para mensuração da espessura cutânea em indivíduos sãos e com sequelas de queimadura, demonstrou ser um método vantajoso, por ser aplicável em qualquer parte do corpo, não utilizar radiação, ter baixo custo, alta reprodutibilidade e baixa variabilidade entre observadores ${ }^{10-14}$.

Em nossa instituição, a ultrassonografia de partes moles foi realizada com o aparelho ATL Philips HD 5000 (Milwalkee - USA), com sonda de pequenas partes de 14 $\mathrm{MHz}$.

Dois grupos de pacientes foram submetidos a preenchimento de sulco nasogeniano (SNG) com duas formulações de ácido hialurônico de um mesmo fabricante, com diferentes pesos moleculares. No primeiro grupo, com peso molecular de 800.000 daltons, houve menor permanência do produto quando comparado ao grupo que recebeu a injeção da fórmula com peso molecular 1.550.000 daltons, após 6 meses. Os resultados confirmaram que a ultrassonografia é método de avaliação eficiente, objetivo e não-invasivo para o estudo de preenchimentos faciais, e levou o fabricante do produto a substituir a fórmula de menor peso molecular pela nova formulação ${ }^{15}$.
O objetivo do presente estudo foi avaliar a durabilidade da formulação atual de ácido hialurônico (Derma Hyal®) 12 meses após preenchimento de SNG, comparando a percepção do efeito clínico com a espessura de partes moles medida à ultrassonografia.

\section{MÉTODO}

Foram selecionadas dez pacientes com idades entre 38 e 55 anos (média 49,1), com queixa de acentuação do SNG por envelhecimento facial. Foram excluídas pacientes com tratamentos ambulatoriais ou cirúrgicos prévios no local, doença sistêmica não controlada e alergia a qualquer componente do produto utilizado. Todas as pacientes assinaram Termo de Consentimento Informado aprovado pela instituição.

Foi utilizado ácido hialurônico a 3\% (Derma Hyal ${ }^{\circledR}$ Oft Vision, São Paulo, Brasil) de peso molecular 1.550.000 daltons, em injeção intradérmica profunda, $1 \mathrm{ml} \mathrm{em} \mathrm{cada}$ SNG, sob técnica asséptica.

A ultrassonografia de partes moles foi realizada antes do procedimento, após um, três, seis, nove e doze meses, além de fotografias e avaliação subjetiva da satisfação. O exame foi realizado sempre pelo mesmo radiologista, com a cabeça da paciente inclinada a $45^{\circ}$, aferindo a espessura da camada dermo-epidérmica em ponto padronizado previamente no $\mathrm{SNG}$, a $1,0 \mathrm{~cm}$ da inserção da asa nasal no mesmo. Em cada momento de avaliação, foram realizadas três medidas de cada lado da face da paciente, utilizando-se como valor final a média das três.

A avaliação quanto à satisfação da paciente diante do procedimento foi mensurada por meio de escore: 0 - insatisfatório (sem diferença quanto ao pré-tratamento), 1 - pouco satisfatório (pouca melhora), 2 - satisfatório, 3 - muito satisfatório (Figuras 1 e 2).

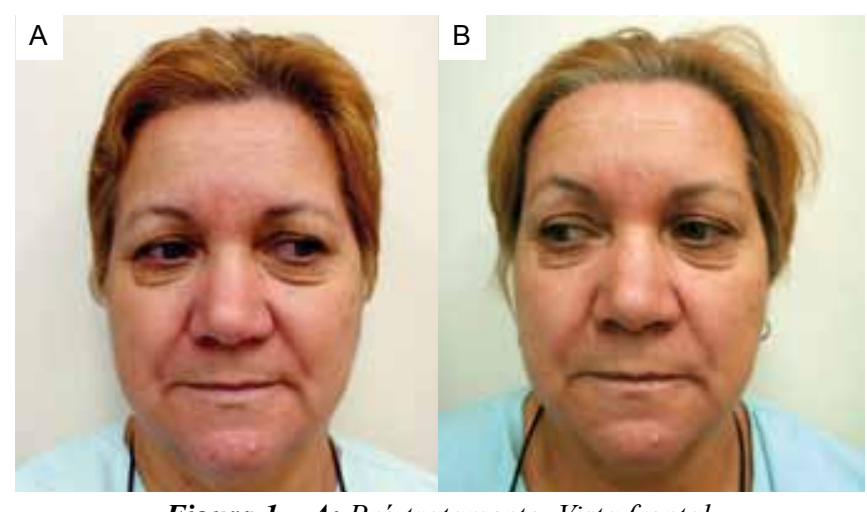

Figura 1 -A: Pré-tratamento. Vista frontal. B: Pós-tratamento - 6 meses. 


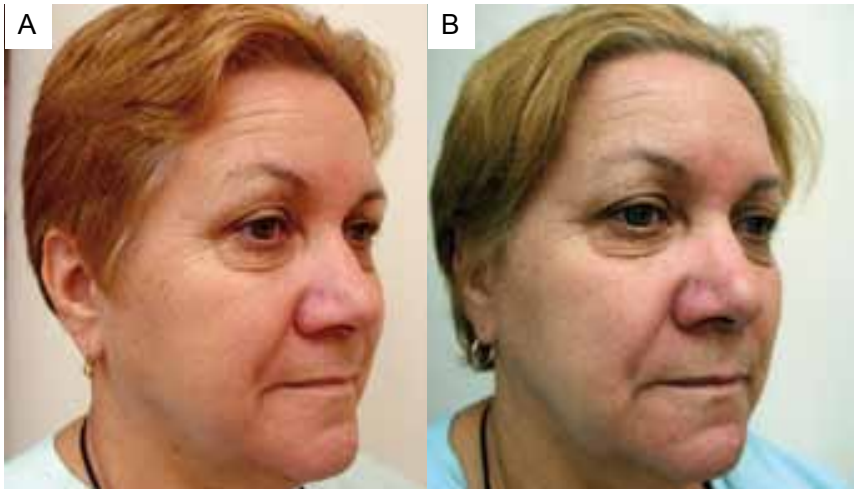

Figura 2-A: Pré-tratamento. Perfil obliquo direito. B: Pós-tratamento - 6 meses.

\section{RESULTADOS}

Dentre as 10 pacientes tratadas, apenas $1(10 \%)$ apresentou hematoma localizado unilateral e $5(50 \%)$, edema leve após o procedimento, todas acompanhadas conservadoramente, com resolução completa em até 10 dias.

Os dados obtidos foram analisados por meio do teste não-paramétrico de Friedman, com objetivo de comparar os resultados da ultrassonografia entre os diversos momentos do tempo. O nível de significância foi de 0,05 (5\%) e os intervalos de confiança foram de $95 \%$.

A média da espessura dos $20 \mathrm{SNG}$ analisados foi de 0,38 $\pm 0,14$ no pré-tratamento, $0,69 \pm 0,19$ após 1 mês, $0,65 \pm 0,17$ após 3 meses, 0,61 $\pm 0,22$ após 6 meses, $0,57 \pm 0,23$ após 9 meses e $0,55 \pm 0,14$ após 12 meses (Figura 3 ).

Das 10 pacientes, 3 avaliaram o resultado aos 6 meses como insatisfatório, 5 como pouco satisfatório e 2 como satisfatório. Apenas uma paciente considerou o tratamento satisfatório após 9 meses.

Aos 12 meses, uma paciente considerou o resultado pouco satisfatório, e as demais consideraram insatisfatório, ou seja, sem diferença em relação ao pré-tratamento. Todas responderam que realizariam novamente o procedimento (Figura 4).

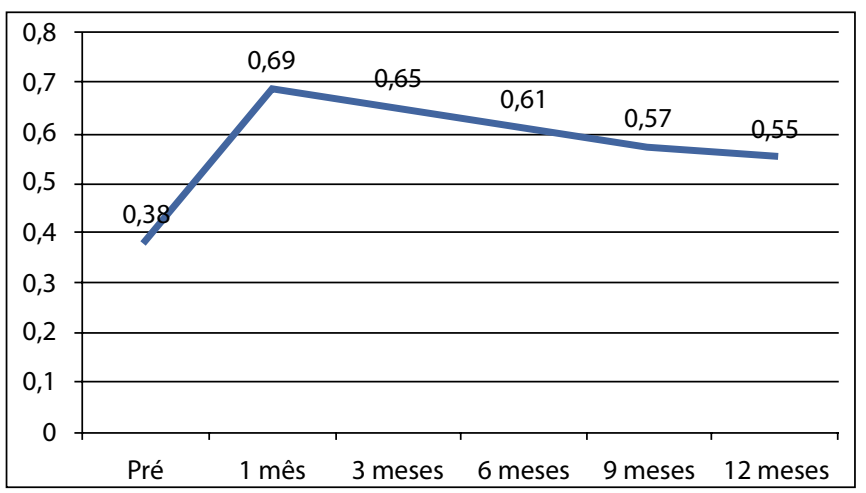

Figura 3 - Média da espessura cutânea do sulco nasogeniano à ultrassonografia, ao longo do tempo (em milímetros).

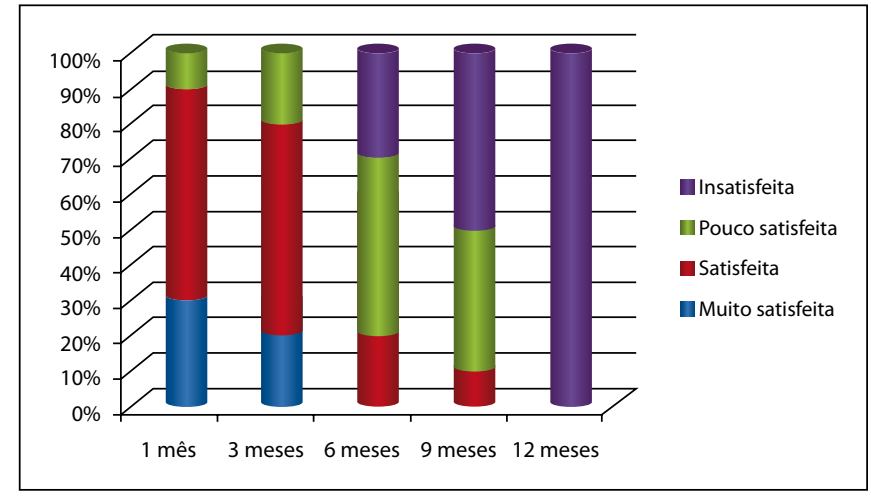

Figura 4 - Avaliação da satisfação das pacientes ao longo do tempo.

\section{DISCUSSÃo}

O envelhecimento cutâneo divide-se em intrínseco ou "cronológico", com início ao redor dos 30 anos e envelhecimento extrínseco ou "fotoenvelhecimento". O primeiro, por causar atrofia dos componentes cutâneos, caracteriza-se por pele de aparência fina, frágil, inelástica, flácida, ressecada, com déficit de reparação após lesões e predisposição ao aparecimento de neoplasias benignas. O envelhecimento extrínseco, principalmente ocasionado pela radiação ultravioleta, apresenta alterações superimpostas que levam a aspereza e ressecamento da pele, teleangiectasias, rítides de maior profundidade e tumores cutâneos, incluindo as neoplasias malignas ${ }^{16}$.

As rugas dividem-se em ortostáticas, dinâmicas, estáticas e gravitacionais, sendo estas últimas originadas pelo descenso dos tecidos a partir de pontos de aderência, como é o caso do $\mathrm{SNG}^{16}$.

Cada vez mais, um número maior de procedimentos ambulatoriais, não invasivos ou minimamente invasivos, vem colaborando para o tratamento e suavização dos diversos tipos de rítides faciais. O ácido hialurônico é a substância absorvível de maior experiência na prática clínica para preenchimento de rugas e sulcos ${ }^{3-6,8-10}$. Há diversas apresentações no mercado, e a durabilidade do efeito clínico tem variado entre elas, sem trabalhos científicos comparativos ${ }^{8}$.

Outras opções de tratamento no preenchimento de rugas, sulcos e depressões faciais incluem o ácido polilático, que apresenta alto custo e necessita de diversas aplicações, e a lipoenxertia. A lipoenxertia tem como principais vantagens a utilização de material autólogo e a possibilidade de utilização de maior quantidade de material em relação aos demais preenchedores ${ }^{17}$. Suas desvantagens incluem a necessidade de realização em ambiente cirúrgico, algum grau de imprevisibilidade dos resultados por conta do grau de absorção e da possibilidade de complicações como infecção, embolia, formação de cistos, calcificações, ossificação, pseudotumores, amaurose, liponecrose, necrose cutânea, hipercorreções, assimetrias, entre 
outros. Além disso, o procedimento tem como fator limitante principal o peso corporal do paciente ${ }^{18,19}$.

Este estudo avaliou a durabilidade do ácido hialurônico Derma Hyal ${ }^{\circledR}$ na concentração 3\%, utilizando ultrassonografia. Com isso, conseguimos demonstrar a permanência do produto na derme profunda ao longo de doze meses, com método objetivo e não-invasivo.

A ultrassonografia tinha sido utilizada previamente na avaliação da eficácia, segurança e durabilidade do ácido polilático em pacientes HIV positivo, com lipoatrofia facial ${ }^{20}$.

Apesar da permanência do aumento de espessura das partes moles observado à ultrassonografia após o sexto mês de tratamento, este achado não se correlacionou com a percepção de efeito clínico pelas pacientes. Os escores dados pelas pacientes refletiram o descontentamento das mesmas, e foram correspondentes à percepção subjetiva da equipe médica, apesar de esta não ter sido registrada por não ser o objetivo do estudo. Esse descontentamento pode ser explicado pela alta expectativa em relação aos resultados de tratamentos de rejuvenescimento facial. Melhores resultados de rejuvenescimento são obtidos por meio da associação de diversos tratamentos, entre eles toxina botulínica, tratamento de pele e reposição de volume facial. O domínio deste conhecimento pelo cirurgião plástico é fundamental para potencializar o resultado das cirurgias faciais.

\section{CONCLUSÕES}

A ultrassonografia demonstrou ser método eficiente, objetivo e não-invasivo na avaliação da durabilidade de preenchimento, desde que avaliado com correlação clínica. Demonstrou-se aumento da espessura cutânea até 12 meses após injeção em SNG, com decréscimo progressivo. Embora exista diferença dos valores da ultrassonografia ao longo do tempo, essas não foram estatisticamente significantes. Clinicamente, o efeito percebido pelas pacientes se deu até ao redor do nono mês pós-aplicação do produto avaliado.

\section{REFERÊNCIAS}

1. Alster TS, West TB. Human-derived and new synthetic injectable materials for soft-tissue augmentation: current status and role in cosmetic surgery. Plast Reconst Surg. 2000;105(7):2515-25.

2. Brody HJ. Use of hyaluronidase in the treatment of granulomatous hyaluronic acid reactions or unwanted hyaluronic acid misplacement. Dermatol Surg. 2005;31(8 Pt 1):893-7.

3. Piacquadio D, Jarcho M, Goltz R. Evaluation of hylan b gel as a soft-tissue augmention implant material. J Am Acad Dermatol. 1997;36(4):544-9.

4. Duranti F, Salti G, Bovani B, Calandra M, Rosati ML. Injectable hyaluronic acid gel for soft tissue augmentation. A clinical and histological study. Dermatol Surg. 1998;24(12):1317-25.

5. Olenius M. The first clinical study using a new biodegradable implant for the treatment of lips, wrinkles, and folds. Aesthetic Plast Surg. 1998;22(2):97-101.

6. Lowe NJ, Maxwell A, Lowe P, Duick MG, Shah K. Hyaluronic acid skin fillers: adverse reactions and skin testing. J Am Acad Dermatol. 2001;6(45):930-3.

7. Cox SE. Clinical experience with filler complications. Dermatol Surg. 2009;35(Suppl 2):1661-6.

8. Alster TS, West TB. New options for soft tissue augmentation. Skin Aging 1998;32-6.

9. Larsen NE, Pollak CT, Reiner K, Leshchiner E, Balazs EA. Hylan gel biomaterial: dermal and immunologic compatibility. J Biomed Mar Res. 1993;27(9):1129-34.

10. Alexander H, Miller DL. Determining skin thickness with pulsed ultrasound. J Invest Dermatol. 1979;72(1):17-9.

11. Tan CY, Statham B, Marks R, Payne PA. Skin thickness measurement by pulsed ultrasound: its reproducibility, validation and variability. $\mathrm{Br}$ J Dermatol. 1982;106(6):657-67.

12. Kirsch JM, Hanson ME, Gibson JR. The determination of skin thickness using conventional diagnostic ultrasound equipment. Clin Exp Dermatol. 1984;9(3):280-5.

13. Van den Kerckhove E, Staes F, Flour M, Stappaerts K, Boeckx W. Reproducibility of repeated measurements on post-burn scars with Dermascan C. Skin Res Technol. 2003;9(1):81-4.

14. Hambleton J, Shakespeare PG, Pratt BJ. The progress of hypertrophic scars monitored by ultrasound measurements of thickness. Burns. 1992;18(4):301-7.

15. Salles AG, Remigio AFN, Saito OC, Camargo CP, Zacchi VB, Saito PL, et al. Avaliação da durabilidade de preenchimento de ácido hialurônico com ultra-som facial. Arq Cat Med. 2009;38(supl 1):281-3.

16. Borelli S, Pellegrino DG, Mapeli AB. O envelhecimento da face: como e por quê envelhecemos. In: Melega JM, Baroudi R, eds. Cirurgia Plástica Fundamentos e Arte: cirurgia estética. Rio de Janeiro:Medsi;2003. p.915.

17. Coleman SR. Structural fat grafting. $1^{\text {a }}$ ed. St. Louis:QMP;2004.

18. Khawaja HA, Hernández-Pérez E. Fat transfer review: controversies, complications, their prevention, and treatment. Intern J Cosm Surg Aesth Dermatol. 2002;4(2):131-8.

19. Lam SM, Glasgold RA, Glasgold MJ. Limitations, complications, and long-term sequelae of fat transfer. Facial Plast Surg Clin North Am. 2008;16(4):391-9.

20. Valantin MA, Aubron-Olivier C, Ghosn J, Laglenne E, Pauchard M, Schoen H, et al. Polylactic acid implants (New-Fill) to correct facial lipoatrophy in HIV-infected patients: results of the open-label study VEGA. AIDS. 2003;17(17):2471-7.

\footnotetext{
Correspondência para:

Alessandra Grassi Salles

Rua Joaquim Floriano, 466 - cj. 21026 - Itaim - São Paulo, SP, Brasil - CEP 04534-002

E-mail: agsalles@uol.com.br
}

Nota: Nenhum dos autores tem qualquer vínculo ou recebe qualquer tipo de benefício das empresas fabricantes e distribuidoras dos produtos utilizados. Os produtos utilizados foram doados pela empresa distribuidora para a realização da pesquisa. 